\title{
Airborne pollution and pregnancy
}

\author{
E Jauniaux ${ }^{a}$ and J Grigg ${ }^{b}$ \\ aEGA Institute for Women's Health, Faculty of Population Health Sciences, \\ University College London, London, UK
}

${ }^{b}$ Centre for Genomics and Child Health, Queen Mary University of London, London, UK

Linked article: This is a mini commentary on T Michikawa et al. To view this article visit http://dx.doi.org/10.1111/1471-0528.15999

There is mounting epidemiological evidence that maternal exposure to air pollution is associated with adverse birth outcomes and in particular with preterm birth (Siddika et al. Environ Res 2019;176:108549). Air pollution results mainly from the combustion of fossil fuels and from industrial emissions and has become a major public health issue for all age groups around the world. Air pollution is a complex mixture of solid and liquid particles. Nitrogen dioxide $\left(\mathrm{NO}_{2}\right)$, sulphur dioxide $\left(\mathrm{SO}_{2}\right)$, carbon monoxide $(\mathrm{CO})$ and inhalable particulate matters $\left(\mathrm{PM}_{2.5}\right.$ or $\left.\mathrm{PM}_{10}\right)$ that contain lead and arsenic have been shown to be strongly associated with chronic obstructive pulmonary disease and lung cancer in adults, and with asthma and acute respiratory infections in children (Liu and Grigg BMJ Paediatr Open 2018;2:e000210).

Exposure of pregnant women has been linked not only to premature birth but also to fetal growth restriction, stillbirth and congenital anomalies of the cardiovascular system (Jacobs et al. Sci Total Environ 2017;579:1179-92). Maternal exposure to air pollution has also been linked to decreased lung function in infancy and childhood, increased respiratory symptoms, and the development of childhood asthma (Korten et al. Paediatr Respir Rev 2017;21:36-6).

Asian dust is a meteorological phenomenon, which often originated from the Gobi desert of Central and East Asia but has been recently exacerbated by human activities, in particular agriculture and mining (Sternberg and Edwards Int $J$ Environ Res Public Health 2017;14:E1342). Particulates trapped in dust clouds increase pollution not only locally but also across continental China, the Korean Peninsula, Japan and the North Pacific Ocean. Asian dust events have been found to be significantly associated with acute myocardial infarction among high-risk Japanese non-smoking men (Kojima et al. Eur Heart $J$ 2017:38:3202-8). The current multiarea study by Michikawa et al. (BJOG 2019; https://doi.org/10.1111/14710528.15999) indicates that Asian dust is associated with an increased risk of placental abruption.

The mechanisms by which prenatal air pollution affects fetal and placental development are not fully understood, but probably involve co-interaction between oxidative-stress-induced telomere-mitochondrial aging, epigenetics and environmental factors (Saenen et al. Clin Epigenetics 2019;11:124). A recent study has found black carbon particles in villous tissue, indicating that the human placental barrier is permeable to airborne particulates (Bove et al. Nat Commun 
2019;10:3866). There is also preliminary evidence that similar black carbon particles can be found in placental and lung macrophages (Liu et al. Eur Respir $J$ 2018;52:PA360) (Figure 1).

Michikawa et al. suggest that the intermediate association between Asian dust and placental abruption could be maternal asthma. However, it should also be noted that exposure to air pollution during pregnancy increases the risk of pregnancyinduced hypertensive disorders, which have a well-established association with placental abruption (Pedersen et al. Hypertension 2014;64:494-500). In addition, active and passive maternal exposures to tobacco smoke are associated with similar effects on the placenta, though many epidemiological studies do not include accurate data on this essential confounder. In particular, most studies rely on questionnaires, which are unreliable; the risks may also vary during pregnancy, according to changes in smoking habits. Adjustment for smoking and other potential confounders, such as pre-eclampsia, are essential to better evaluate the impact of air pollution on pregnancy outcomes.

\section{Disclosure of interests}

Both authors are scientific advisers to the Committee on the Medical Effects of Air Pollutants (COMEAP), subgroup on the effects of maternal exposure to ambient air pollution on maternal birth outcomes, Public Health England. Completed disclosure of interest forms are available to view online as supporting information. 
Figure 1 Light microscopy of an airway macrophage with similar degree of loading of black carbon to placental macrophages.

\section{$10 \mu \mathrm{m}$}

